\title{
Lumen
}

Selected Proceedings from the Canadian Society for Eighteenth-Century Studies

\section{La plume et les Lumières : le manuscrit, outil de progrès}

\section{Geneviève Artigas-Menant}

Volume 19, 2000

Material Productions \& Cultural Construction

Culture matérielle \& Constructions discursives

URI : https://id.erudit.org/iderudit/1012312ar

DOI : https://doi.org/10.7202/1012312ar

Aller au sommaire du numéro

Éditeur(s)

Canadian Society for Eighteenth-Century Studies / Société canadienne d'étude du dix-huitième siècle

ISSN

1209-3696 (imprimé)

1927-8284 (numérique)

Découvrir la revue

Citer cet article

Artigas-Menant, G. (2000). La plume et les Lumières : le manuscrit, outil de progrès. Lumen, 19, 1-21. https://doi.org/10.7202/1012312ar

Copyright (c) Canadian Society for Eighteenth-Century Studies / Sociéte canadienne d'étude du dix-huitième siècle, 2000
Ce document est protégé par la loi sur le droit d'auteur. L'utilisation des services d'Érudit (y compris la reproduction) est assujettie à sa politique d'utilisation que vous pouvez consulter en ligne.

https://apropos.erudit.org/fr/usagers/politique-dutilisation/ 


\section{La plume et les Lumières: le manuscrit, outil de progrès}

'Cet art si favorable à l'avancement des Sciences, qui acquièrent toujours de la perfection à mesure que les connaissances se multiplient, fut trouvé vers le milieu du 15ème siècle.' Le début de l'article 'imprimerie' de l'Encyclopédie associe 'l'avancement des sciences' à la découverte de Gutenberg. L'auteur poursuit:

non seulement l'on est parvenu rapidement, par le secours de l'impression, à multiplier les connaissances, mais encore à fixer et à transmettre jusqu'à la fin des siècles les pensées des hommes, tandis que leurs corps sont confondus avec la matière, et que leurs âmes se sont envolées au séjour des esprits.

Tous les autres arts qui servent à perpétuer nos idées périssent à la longue. Les statues tombent finalement en poussière. Les édifices ne subsistent pas aussi longtemps que les statues, et les couleurs durent moins que les édifices. Michel Ange, Fontana et Raphaël sont ce que Phidias, Vitruve, et Appelle étaient à la sculpture, et les travaux de ceux-ci n'existent plus. L'avantage que les auteurs ont sur ces grands ancêtres vient de ce qu'on peut multiplier leurs écrits, en tirer, en renouveler sans cesse le nombre d'exemplaires qu'on désire, sans que les copies le cèdent en valeur aux originaux ... Puisqu'il est vrai que les livres passent d'un siècle à l'autre, quel soin ne doivent pas avoir les auteurs d'employer leurs talents à des ouvrages qui tendent à perfectionner la nature humaine?

Le message de l'encyclopédiste est clair: depuis le milieu du XVe siècle, l'avenir de l'écrit est dans l'impression; écrivons pour être imprimés, c'est le moyen d'assurer le progrès humain.

$C^{\prime}$ est bien en effet selon ce schéma traditionnel du progrès fondé sur le développement de l'imprimerie que nous envisageons les Lumières. Dans la deuxième moitié du dix-huitième siècle, les emprunts, les investissements de capitaux augmentent les achats de presses et de papier, propulsent l'imprimerie du stade artisanal au stade capitaliste et industriel, entraînent la baisse des coûts, favorisent la diffusion massive des connaissances et des idées, donc le progrès de l'esprit. Depuis Guten- 
berg, on pouvait imprimer; trois siècles plus tard, grâce aux nouvelles performances de l'imprimerie et de la librairie, aux Lebreton, Panckoucke, Cramer, Marc Michel Rey, on peut imprimer vite, imprimer plus, imprimer tout.

Le Dictionnaire des Sciences et des Arts, symbole des Lumières et entreprise de librairie, est la démonstration de ce grand mouvement. Sans les capitaux de Lebreton, point d'Encyclopédie, de frontispice, de 'Vérité enveloppée d'un voile, et rayonnante d'une lumière qui écarte les nuages et les disperse, ${ }^{1}$ de Raison et de Philosophie faisant plier le genou à la Théologie, selon les termes de la légende de la gravure de Cochin. Tout cela est vrai, mais en partie seulement.

Depuis la fin du XVIIe siècle, éclairés par la raison et la philosophie, des écrivains laborieux fabriquent les arguments de la lutte antichrétienne, rédigent les raisonnements, façonnent les commentaires, sans songer à l'impression, et la plume à la main. Des lecteurs curieux annotent, corrigent, composent des recueils, élaborent des extraits, la plume à la main. Des copistes, de métier ou amateurs, reproduisent tout à un grand rythme, traités déistes, athées, hétérodoxes, ou simplement critiques, examens, analyses, dialogues, lettres, entretiens. Des personnages discrets, comme le petit bossu que Voltaire a bien connu, ${ }^{2}$ vendent la marchandise sous le manteau. Des réseaux de colportage la diffusent dans les ballots de livres imprimés. Elle aboutit dans 'le portefeuille de tous les curieux.'

Quelle est l'histoire de ces 'manuscrits philosophiques clandestins?' Comment peut-on expliquer cet étonnant phénomène? Pourquoi, à l'époque de l'imprimerie, le manuscrit est-il un fantastique outil de progrès?

L'histoire fascinante de ces manuscrits bien particuliers se confond presque avec l'histoire de la recherche menée à leur sujet depuis un peu moins d'un siècle, quatre-vingt-six ans exactement. Ils constituent en effet à l'heure actuelle un corpus impressionnant, assez bien délimité, mais ne correspondent pas à une classification de bibliothèque, ni au XVIIIe siècle ni de nos jours. Lorsque l'article 'manuscrit' du Dictionnaire de Trévoux, ${ }^{3}$ dans l'édition de 1752, donnait comme exemple 'cette pièce est défendue, et ne court qu'en manuscrit,' le contemporain appliquait sans doute cette définition très globalement à tout ce qui tombait sous le coup de la censure, dans des domaines aussi divers que satire politique, poésie érotique, débat théologique. Nos manuscrits n'y entrent donc qu'indirectement et pour une très petite part.

Ils font partie, pour leurs contemporains et pour la postérité jusqu'au début du XXe siècle, de la masse impressionnante de manuscrits du XVIIIe siècle qui étaient alors d'un usage quotidien et que conservent aujourd'hui nos bibliothèques. A l'époque des manuscrits que nous 
appelons maintenant 'philosophiques clandestins,' on lit la plume à la main, on pense la plume à la main, on dialogue la plume à la main sans avoir forcément des choses dangereuses à dire. Le manuscrit n'est pas un brouillon, un écrit strictement privé, une forme inférieure de la littérature, une étape avant la publication. La seule façon de diffuser un écrit au XVIIIe siècle n'est pas de l'imprimer. ${ }^{4}$ La copie, gratuite ou lucrative, est une activité permanente, forme de l'activité intellectuelle dont on fait l'apprentissage au collège. On copie des lettres, des poésies, des traductions, des livres de toutes sortes, en entier ou par extraits. On fait des recueils de ces copies, parfois en les mélangeant avec des textes imprimés, annotés ou non. Comment dans ces conditions a-t-on pu identifier et isoler le groupe précis qui nous occupe?

C'est très précisément en 1912 que Gustave Lanson a révélé son existence dans la Revue d'Histoire littéraire de la France. ${ }^{5}$ Sa découverte repose sur la consultation du Catalogue général des manuscrits des bibliothèques publiques de France et sur l'analyse d'une soixantaine de volumes de manuscrits dans diverses bibliothèques, à Paris et en province. ${ }^{7}$ Vingt d'entre eux sont des recueils. Son enquête porte donc sur cent pièces, ou unités, et par le phénomène des copies, sur trente-cinq textes différents. Parmi eux figurent le Mémoire de Jean Meslier, ${ }^{8}$ Le Ciel ouvert à tous les hommes de Pierre Cuppé, ${ }^{9}$ l'anonyme Militaire philosophe, ${ }^{10}$ des ouvrages attribués à Boulainvilliers, Fréret, Dumarsais, Mirabaud. Une telle moisson suffit à lui faire pressentir que c'est 'une erreur, ou du moins une interprétation inexacte des faits' de dater 'le développement de l'incrédulité radicale et violente' $\mathrm{d}$ 'après 1750 . Il fait alors l'hypothèse que

la transformation du mouvement philosophique que la librairie accuse après 1750 pourrait bien se rapporter plutôt à un changement de la tactique qu'à un changement des idées; [qu'] il se pourrait que les volontés après 1750 fussent devenues plus hardies, les passions plus emportées, que les barrières et les digues eussent cédé, et qu'ainsi on eût dès lors publié sans crainte ce qui auparavant se disait à huis clos et tout bas.

Il en conclut que l'histoire de la pensée philosophique avant 1750 passe par l'étude de tels manuscrits et des conditions de leur inspiration, de leur rédaction, de leur diffusion, de leur influence.

Il est important d'insister ici sur la différence de nature entre les textes ainsi repérés et les ouvrages imprimés 'du second et troisième ordre' dont l'historien de la littérature connaissait déjà l'existence et l'oubli. Leur intérêt n'est pas d'apporter quelques nouveaux témoignages isolés de ce qui s'écrivait sans beaucoup de talent à l'époque et autour de Fontenelle, Montesquieu, Voltaire, mais de révéler la 'masse anonyme' 
des manuscrits où 'il faut aller chercher la pensée dangereuse et neuve qui circulait' et 'de laquelle ... se sont élevés' les grands écrivains. Si Lanson présente successivement les auteurs avérés, les auteurs supposés et les textes anonymes les plus remarquables, ce n'est pas pour en célébrer individuellement la pensée mais pour les présenter comme exemplaires du phénomène qu'il veut mettre au grand jour: la constitution de 'tout l'arsenal des arguments critiques, historiques et philosophiques contre la religion et la spiritualité ou l'immortalité de l'âme.' Boulainvilliers n'est pas, affirme-t-il, 'une exception singulière: il est tout au moins chef de choeur et représentatif d'une assez nombreuse catégorie d'esprits.'

Les textes ainsi présentés ont trois caractéristiques essentielles. Premièrement, ce qui les définit, ce qui les isole, ce qui a attiré l'attention de Gustave Lanson, c'est qu'ils sont manuscrits, non pas par accident, mais par nature en quelque sorte, c'est-à-dire par opposition aux textes imprimés qu'ils précèdent dans le siècle; c'est sous la forme manuscrite qu'ils ont exercé leur influence sur l'histoire des idées. Deuxièmement ils sont 'philosophiques' au sens large des Lumières, c'est-à-dire critiques, hostiles à 'tout l'appareil de dogme, d'histoire et de philosophie sur lequel le christianisme repose.' Enfin, ils sont clandestins; le mot n'apparaît que plus tard ${ }^{11}$ mais l'idée vient de Lanson qui met l'accent sur la stratégie de la diffusion discrète dans un temps et un pays où 'la liberté d'imprimer [n'est pas ] entière. ${ }^{, 12}$ L'idée qu'ils forment véritablement un ensemble, qu'il faut les analyser bout à bout, les comparer entre eux parce qu'ils constituent un corpus dont la signification réside dans sa globalité même, cette idée est la découverte que Lanson a léguée aux chercheurs du XXe siècle. Des questions se posent alors sur les rapports à l'intérieur de cette famille de manuscrits. S'agit-il d'influences de l'un sur l'autre? et dans quel sens? ou bien de l'usage commun des mêmes arguments? Quelles sont les 'sources de l'érudition et de la critique de ces incrédules?' Qu'ont-ils transmis à leurs successeurs? Pour y répondre Gustave Lanson lance très méthodiquement un programme de recherche qui tient en deux points complémentaires: faire une 'étude exacte des nombreux manuscrits philosophiques du XVIIIe siècle que possèdent les bibliothèques des départements' français et dresser 'une liste des copies conservées, en essayant d'établir l'époque de leur fabrication, ou de la fabrication de l'original dont elles dérivent,' qui sera le moyen de 'conjecturer, d'après le nombre des copies, la diffusion possible des idées.' ${ }^{13}$

Il faut attendre 1938 pour voir le premier ouvrage qui commence à remplir ce double programme. L'érudit américain Ira $\mathrm{O}$. Wade applique à la lettre les consignes de Lanson. ${ }^{14}$ Comme il le dit lui-même, nombreuses sont les savantes études, y compris de grandes synthèses comme celles de Daniel Mornet et Paul Hazard, ${ }^{15}$ qui ont paru depuis 1912, mais 
il est le premier à considérer les 'questions diverses sur l'histoire de l'esprit philosophique en France avant 1750,' selon le titre de l'article de Lanson, comme de vraies questions et à tenter d'y répondre. Il poursuit l'investigation à Paris, l'étend à quarante et une villes de province et pratique des sondages à l'étranger. Le résultat est stupéfiant: il dresse une liste de cent deux titres, soit soixante-sept de plus que son prédécesseur, auxquels correspondent en tout quatre cent trois copies, soit deux cent quatre vingt treize de plus. Un tel résultat confirme, au-delà de tout espoir, les intuitions de Lanson sur la diffusion manuscrite et donne une preuve irréfutable de la réalité du groupe de manuscrits qu'il désignait.

Cela est tellement vrai qu' on peut dire maintenant qu'on n'arrête pas de trouver dans le monde entier de nouveaux manuscrits qui étendent la famille découverte par Lanson. Depuis 1980, le chercheur espagnol Miguel Benítez met à jour et publie régulièrement des listes qui associent les trouvailles individuelles et dispersées aux listes initiales de Lanson et Wade. Les chiffres sont parlants. En 1980, la liste passait de cent deux titres à cent trente, en 1988 à cent quarante huit. En 1996, elle comptait cent quatre vingt douze titres pour près de deux mille copies. ${ }^{16}$ En outre, depuis 1992 une publication annuelle, La Lettre clandestine, ${ }_{1}^{17}$ fait le point régulièrement sur les nouvelles découvertes.

Les manuscrits ainsi mis au jour se sont peu à peu intégrés au paysage intellectuel des Lumières, en le transformant, et les chercheurs ont été de plus en plus nombreux, à partir de 1950, non seulement à tenir compte de leur existence mais à aborder les questions qu'ils soulèvent, sur l'origine des idées qu'ils véhiculent, sur les traditions auxquelles ils se rattachent, sur les sources et modèles étrangers, sur les rapports avec la pensée réformée, avec les controverses internes au catholicisme, avec le libertinage érudit, sur leur réception, leur influence, la transformation de leurs matériaux en oeuvres littéraires, leur place dans l'histoire de la librairie, etc. Il ne faudrait surtout pas croire que ces travaux relèvent d'un intérêt préconçu pour les oeuvres mineures. Il ne s'agit absolument pas d'étudier par principe les auteurs secondaires, après les grands, ou à côté des grands, mais de tenir compte d'un fantastique mouvement d'idées, peu voyant, mais très actif.

Le développement de ce domaine de recherche longtemps inconnu est spectaculaire. ${ }^{18}$ Les chercheurs se heurtent à deux difficultés principales. La première, on l'aura deviné tient à la constante évolution quantitative, du corpus. La seconde, sur laquelle je reviendrai plus longuement, tient à une particularité dont il faut dire un mot ici. On ne sait jamais à distance ce qui se cache sous un titre. Le même titre peut avoir été donné à deux textes totalement différents. Deux textes parfaitement semblables peuvent se dissimuler sous des titres assez ou très différents. 
La littérature qui nous occupe est une matière toujours vivante, sujette à toutes les transformations possibles. Tant qu'il circule sous forme manuscrite, un texte n'est jamais définitivement fixé. Chaque nouvelle copie peut être l'occasion d'une nouvelle version. Dans l'absolu, pour travailler sur un texte clandestin, même s'il a été édité, il faudrait avoir une connaissance personnelle de tous les exemplaires manuscrits, intégraux ou abrégés, qui en existent. La dispersion des manuscrits, de plus en plus grande au fur et à mesure que le recensement avance, rend ces conditions parfaites irréalisables dans la plupart des cas. L'idéal serait donc de disposer d'une sorte d'inventaire descriptif et analytique de tous les textes déjà repérés.

C'est dans l'intention de le constituer et de le publier qu'Olivier Bloch, professeur d'Histoire de la philosophie à Paris I, a créé en 1987 l'équipe de $1^{\prime}$ Inventaire des manuscrits philosophiques clandestins, ${ }^{19}$ composée de chercheurs et d'enseignants-chercheurs en histoire, littérature et philosophie, ainsi que de conservateurs des bibliothèques. Notre travail est presque terminé dans les bibliothèques parisiennes, irrégulièrement avancé dans les provinces françaises, selon les régions. Le premier volume, consacré à la Bibliothèque Mazarine, est prêt pour la publication. Quarante-trois collaborateurs, de huit pays différents, de l'Argentine à la Finlande, y ont travaillé.

Le développement accéléré de la recherche, surtout depuis les années 70, sur un objet inconnu avant 1912 s'accompagne, on s'en doute, de passionnants débats. Le plus ardent porte sur l'interprétation du phénomène découvert: comment expliquer que pendant plus de soixante ans une si fantastique machine à diffuser les idées dangereuses sous forme manuscrite ait fonctionné si activement?

La présentation rapide et nécessairement trop schématique que je viens de faire des manuscrits philosophiques clandestins et de leur découverte contient implicitement une première réponse. C'est l'interprétation que Lanson suggère lui-même dans son article fondateur sans entrer dans le détail parce qu'il n'en voit qu'une et qu'elle lui paraît suffisante. Son intention première était $\mathrm{d}^{\prime \prime}$ 'écrire l'histoire du mouvement philosophique en France au XVIIIle siècle.' Il s'aperçoit chemin faisant qu'elle est loin d'être faite et que les données essentielles font encore défaut parce qu'il découvre l'existence d'une masse de textes critiques aussi audacieux, aussi violents, aussi radicaux que ceux de Voltaire, de Diderot, des Encyclopédistes, de D'Holbach et Naigeon. Il peut dater les plus importants. Le Mémoire de Meslier est forcément antérieur à 1729, année de sa mort. Boulainvilliers est mort en 1722, les manuscrits qu'on lui attribue sont apparemmment antérieurs, le manuscrit 1163 de la Bibliothèque Mazarine intitulé Difficultés sur la religion proposées au père Malebranche, est l'original du Militaire philosophe 
publié par D'Holbach en 1768. Son contenu nous apprend qu'il a été écrit vers 1710. Pierre Cuppé a forcément écrit Le Ciel ouvert avant 1716, année de sa rétractation. Ils sont presque tous restés manuscrits jusqu'à une date tardive dans le siècle parce qu'il aurait été dangereux de les publier plus tôt. Il existe plusieurs copies de tous ces textes, parfois en très grand nombre, donc ils ont circulé et ont pu influencer ceux qu'on appelle les philosophes des Lumières. Ces manuscrits ont préparé la révolution intellectuelle de la fin de l'Ancien Régime, ils sont, dans ce sens, outils de progrès. En 1938, lorsqu'il décrit, c'est son titre, 'l'organisation et la diffusion clandestine des idées philosophiques en France de 1700 à 1750,' Wade reprend totalement à son compte cette interprétation.

Elle a le mérite de la simplicité et de la globalité. Très rapidement, dès 1939 , avec le compte rendu du livre de Wade par Norman L. Torrey, ${ }^{20}$ elle a rencontré des objections de détail dont aucune ne constitue une remise en cause du phénomène découvert mais qui contribuent toutes à souligner son étonnante complexité. Ainsi peu à peu la réflexion sur les manuscrits philosophiques clandestins s'enrichit de nouvelles explications. La première réticence à l'interprétation primitive, la plus persistante, concerne la chronologie. Il n'est pas facile de dater précisément un manuscrit à l'intérieur du siècle, la mention de la date dans le filigrane du papier français n'étant obligatoire qu'après 1743 et rien n'empêchant d'écrire sur du papier étranger. Lanson et Wade partent du principe que les ouvrages considérés ayant été écrits tôt dans le siècle, leur diffusion est elle aussi intervenue tôt. Leurs contradicteurs signalent que certains recueils écrits de la même main réunissent des copies d'ouvrages de Voltaire postérieurs à 1760 avec des copies d'écrits beaucoup plus anciens. Ils remarquent aussi que rien ne prouve que beaucoup de copies n'ont pas été faites à partir d'une édition postérieure à 1750, lorsqu'elle existe. Il est vrai que les Archives de la Bastille prouvent qu'il circulait des manuscrits tôt dans le siècle, que les témoignages prouvent l'existence du petit bossu de Voltaire ${ }^{21}$ et de ses comparses qui vendaient les manuscrits sous le manteau dans la première moitié du siècle. Il n'est pas question de le nier, mais, disent-ils, rien ne prouve que la masse impressionnante des copies manuscrites est majoritairement précoce et que les ouvrages du début du siècle ont connu de bonne heure une ample diffusion manuscrite. L'influence des manuscrits philosophiques de la première moitié du siècle sur les ténors de la seconde moitié n'est peut-être pas si grande que Lanson l'a cru.

Ces observations justifiées apportent des nuances plus que de vraies contradictions et ne changent rien aux données fondamentales. Si l'on ignore la juste proportion de copies qui ont été faites avant 1750 et de copies qui ont été faites après, de copies qui ont été faites sur une édition et de copies qui ont été faites sur un manuscrit, il n'en demeure pas moins 
que la masse totale de manuscrits prouve leur intense diffusion. Que celle-ci se soit produite surtout avant ou surtout après l'impression, lorsqu'elle a eu lieu, ne modifie pas le fond du problème. En ce qui concerne le plus ou moins grand degré d'influence des manuscrits sur la pensée des Lumières, comment, dans tous les cas, prétendre le déterminer avec précision? Le problème d'ensemble reste posé mais se complique d'une certaine façon: il faut trouver une explication qui rende compte de la diffusion manuscrite dans tout le siècle et non plus seulement dans sa première moitié.

L'édition, la vente, la circulation des livres sont étroitement surveillées. La censure fonctionne. La police veille. La justice punit. La Bastille fait peur aux auteurs, aux imprimeurs, aux libraires. Les Archives de la police attestent des fouilles et des arrestations. Mais la sévérité des règlements varie dans le siècle, leur efficacité est toujours incertaine, l'imprimerie ne chôme pas, même avant 1750, les livres prohibés circulent et lorqu'ils sont persécutés, ils ne cessent pas totalement de circuler. Si l'on a vraiment envie de faire imprimer un brûlot d'irréligion, les moyens ne manquent pas. Le livre clandestin se porte bien et circule beaucoup. ${ }^{22}$ On peut imprimer à l'étranger ou même en France à certaines conditions. Personne ne nourrissait d'illusion sur l'origine française de ces éditions. Un article des Mémoires de Trévoux ${ }^{23}$ daté de mars 1705 à Rennes donne l'information suivante: 'il s'est répandu depuis peu une traduction d'une Lettre d'Hippocrate à Damagette imprimée à Cologne chez Jacques Lesage ... une personne digne de foi nous assure que cet ouvrage plein d'une impiété grossière a été fabriqué auprès de Rouen.' Le même journal commente en août 1743 la sortie d'un recueil imprimé sous l'adresse d'Amsterdam, intitulé Nouvelles Libertés de penser ${ }^{24}$ :

tous ces petits livres se font au gré de certains petits libraires par de petits auteurs qui savent bien tous qu'on gagne quelque argent à flatter les passions d'un certain petit public .... On sait que tous ces petits livres pareils à celui-ci, sont l'effet de l'avarice des libraires et de l'indigence des auteurs et qu'il ne tient qu'à un libraire de Hollande ou d'Angleterre de tourner par quelques schellings ou florins de plus ou de moins ces plumes mercenaires pour ou contre la religion, à leur gré.

Certes un manuscrit est plus clandestin qu'un livre imprimé avec permission et privilège mais un manuscrit est-il forcément plus clandestin qu'un livre sans autorisation de la censure, imprimé à Londres ou Amsterdam, ou en France sous une fausse adresse? La question n'est pas du plus ou moins grand degré de clandestinité du livre ou du manuscrit, ce qui compte c'est que le manuscrit soit resté manuscrit alors qu'il 
pouvait, comme d'autres, devenir imprimé clandestin. C'est le cas de cette fameuse Traduction de la Lettre d'Hippocrate à Damagette publiée en 1700 dont parlent les Mémoires de Trévoux, pour ne citer que cet exemple passionnant parce qu'on n'en connaît pas moins de quatorze copies manuscrites. Un autre cas retient l'attention, c'est celui de La Réponse en forme de dissertation à un théologien qui demande ce que veulent dire les Sceptiques, qui cherchent la vérité, par le sieur Gaultier, médecin à Nyort, édité à Nyort, Chez Jean Elies, Imprimeur et marchand libraire en 1714. Ce livre imprimé est l'original d'où a été tiré un traité clandestin, Parité de la vie et de la mort, actuellement connu sous la forme de deux manuscrits et d'un pièce imprimée dans un recueil sans date qu'on peut situer autour de 1771. Les quatre exemplaires de La Réponse du médecin Gaultier présentent tous les caractères d'une édition officielle, approbations et permis d'imprimer. Comme on ne relève aucune trace de supercherie, il faut donc croire que ce traité clairement matérialiste a vu le jour avec la bénédiction des autorités deux ans avant la fin du règne de Louis XIV. ${ }^{25}$ Il est clair qu'on ne peut s'en tenir, pour expliquer les manuscrits philosophiques clandestins à l'interprétation exclusive par l'absence de liberté d'expression, la prudence et la crainte des poursuites judiciaires.

On voit bien dans tout cela les liens étroits qui unissent l'histoire des manuscrits clandestins à l'histoire du livre et de la librairie. C'est de ce côté-là que viennent des suggestions d'ordre économique et commercial. Je viens de signaler qu'on ne connaissait aujourdhui que quatre exemplaires de l'imprimé officiel et matérialiste de 1714. Il n'est pas rare que nous ne connaissions même qu'un exemplaire imprimé d'un texte et parfois nous savons qu'une édition a existé et nous n'en possédons aucun exemplaire. C'est vrai pour toutes les sortes de livres au XVIIIe siècle, parce que la réglementation de la librairie limitait le nombre des libraires et imprimeurs et parce que l'impression coûtait cher. Donc en dehors de quelques entreprises florissantes, les imprimeurs en place avaient des moyens réduits, ils imprimaient peu et les livres coûtaient cher. D'où la copie manuscrite qui ne se limitait pas, je l'ai déjà dit, aux traités philosophiques tendancieux. Certes, mais cette explication complémentaire n'est pas en proportion avec la masse impressionnante de la famille de manuscrits découverts depuis 1912.

En restant dans l'ordre économique et commercial, on trouve une autre motivation, on pourrait dire la motivation inverse. S'il coûte moins cher de copier soi-même un livre ou de le faire copier par un domestique qui sait écrire que de l'acheter, il est en revanche beaucoup plus lucratif de vendre un livre manuscrit qu'un livre imprimé. Vendu à l'unité et sous le manteau, le manuscrit a un parfum d'interdit, de rareté et de scandale auquel ne résistent pas certains chalands qui trouvent dans l'acquisition et la possession de manuscrits un signe de leur ap- 
partenance à l'élite sociale et intellectuelle. Cet aspect des choses est indéniable mais il ne concerne que les belles copies des professionnels auxquelles ne se réduisent pas, on va le voir, les manuscrits qui nous occupent.

On peut enfin avancer sans risque une hypothèse d'ordre esthétique. La plupart de ces traités, qu'ils soient restés anonymes ou non, sont de laborieuses dissertations dont les auteurs n'étaient pas de grands écrivains et il n'y a pas lieu de déplorer qu'ils soient restés à l'état manuscrit. Lorsqu'enfin certains d'entre eux ont été imprimés, tard dans le siècle, ils l'ont été après de substantiels remaniements. Les chefs-d'oeuvre sont rares en effet dans notre littérature clandestine mais l'impression n'est pas la garantie du talent, ni au XVIIIle siècle ni dans d'autres temps. On approche ici cependant d'une explication essentielle que je développerai dans un instant et qui concerne la conscience intime de nos auteurs qui n'étaient pas des écrivains et qui ne prétendaient pas l'être.

On constate qu'aujourd'hui historiens des idées, historiens de la société et historiens du livre concourent pour proposer des explications du phénomène des manuscrits philosophiques clandestins. Elles sont toutes éclairantes, ne se contredisent pas et sont même remarquables par leur complémentarité. Mais en les additionnant toutes on élucide de plus en plus de points de détail sans aboutir réellement à une vue d'ensemble. Toutes les preuves, historiques, judiciaires, économiques, sociales, esthétiques fournies pour justifier leur statut manuscrit banalisent ces textes pourtant si particuliers. Pour les uns ils ne sont guère que les sources de véritables œuvres des Lumières, pour d'autres des substituts d'imprimés, pour d'autres encore des objets de curiosité parmi d'autres. En somme on accumule une série de vraisemblances sans atteindre à une vérité. Ce que laisse de côté toute cette argumentation, exacte mais fragmentaire, sur les manuscrits philosophiques clandestins, c'est leur originalité massive, indéniable, provocante pour l'esprit.

Une question se pose en effet devant la multiplicité des explications qui corrigent, complètent ou nuancent l'interprétation globale de Lanson et Wade: faut-il définitivement renoncer à une interprétation synthétique du phénomène des manuscrits philosophiques clandestins? On part d'une vision d'ensemble cohérente et indiscutée:

On rencontre dès la fin du XVIIe siècle et dans les premières années du XVIIIe siècle, des négations hautaines, radicales, véhémentes, injurieuses, des négations cuirassées d'érudition et soutenues de science ou de métaphysique, qui heurtent tout l'appareil de dogme, $\mathrm{d}$ 'histoire et de philosophie sur lequel le christianisme repose. Mais ces négations, sauf exception, sont demeurées manuscrites, ou toujours, ou longtemps. Plusieurs des ouvrages les plus hardis ou les plus violents qui furent imprimés après 1750 , de ceux qui nous servent à étudier la 
grande bataille de 1760-1770, datent en réalité du commencement ou du moins de la première partie du XVIIIe siècle. ${ }^{26}$

On complète cette interprétation parce que le corollaire dont elle s'accompagne néglige l'existence des nombreuses copies manuscrites postérieures à 1760. En effet affirmer comme le fait Lanson que 'la résistance du pouvoir aux idées nouvelles remit les choses, pour les novateurs qui n'avaient pas la soif du martyre, en l'état où elle étaient avant l'invention de l'imprimerie,' c'est mettre implicitement l'accent sur la copie et la circulation manuscrite de la première moitié du siècle sans se soucier de la persistance du phénomène. Du coup le rôle des manuscrits philosophiques clandestins dans le progrès des Lumières se dilue dans l'idée $d^{\prime}$ une vague tactique parallèle à l'imprimé sans spécificité parlante.

La pierre d'achoppement dans tout cela c'est la fracture supposée qui disloque le dix-huitième siècle en deux moitiés. De façon paradoxale la découverte des manuscrits philosophiques clandestins l'a d'abord remise en cause, puis, comme par inadvertance, remise en circulation, modifiée mais triomphante. Je m'explique. Lanson et Wade montrent avec beaucoup de discernement que les traités déistes, matérialistes, athées écrits avant 1730 étaient aussi violents, aussi déterminés, aussi destructeurs que ceux qui ont été imprimés après 1750 et que donc cette barrière, cette scission chronologique que dessine l'histoire de la librairie, doit être estompée par l'histoire des idées. Malheureusement ils la rétablissent eux-mêmes d'une autre façon par une vision téléologique: les imprimés de la seconde moitié du siècle seraient l'aboutissement des manuscrits de la première moitié. Alors leurs contradicteurs font tomber à leur tour cette barrière de 1750 en montrant que la diffusion manuscrite a été au moins aussi active après qu'avant, mais ils la reconstruisent aussitôt en concluant que les imprimés d'après 1750 ne doivent sans doute pas tant qu'on pourrait croire aux manuscrits d'avant. Peut-être bien que les uns et les autres ne sont pas loin de la vérité mais les arbres cachent la forêt.

Il faut dire en effet que quelques extraordinaires métamorphoses occupent le devant de la scène. Rappelons les deux cas les plus célèbres dont la symétrie frappante ajoute un attrait piquant au témoignage scientifique.

En 1762, Voltaire publie à Genève, sous le titre d'Extrait des sentiments de Jean Meslier... et de Testament de Jean Meslier deux éditions très expurgées du Mémoire des pensées et sentiments de Jean Meslier sur une partie des erreurs et des abus de la conduite et du gouvernement des hommes .... ${ }^{27}$ Avant de mourir en 1729, Jean Meslier, curé d'Etrépigny en Champagne a écrit ce Mémoire pour témoigner après sa mort de ses vrais sentiments et en a 
fait lui-même trois copies qu'il a déposées au greffe de la justice. Ces trois manuscrits autographes presque identiques sont actuellement conservés à la Bibliothèque Nationale. ${ }^{28}$ Le moins volumineux des trois compte 622 pages in octavo. Ce Mémoire d'une vigueur exceptionnelle est une démonstration divisée en huit énormes chapitres appelés 'preuves de la vanité et de la fausseté des religions.' L'édition de Voltaire fait cinquante neuf pages in octavo. Elle comporte des extraits minuscules des cinq premières preuves. Manquent complètement les trois preuves tirées successivement 'de la tyrannie des grands,' de la 'fausseté même de l'opinion des hommes touchant la prétendue existence des dieux,' de la 'fausseté même de l'opinion que les hommes ont de la spiritualité et de l'immortalité de leurs âmes.' Par de vigoureuses censures Voltaire a défiguré un énorme traité révolutionnaire, matérialiste et athée d'une sincérité étonnante, en abrégé déiste méconnaissable mais plus facile à lire.

En 1767, paraît sous la date de 1768 et à l'adresse de Londres, mais en réalité à Amsterdam chez Marc-Michel Rey, un ouvrage anonyme de cent quatre-vingt-treize pages intitulé Le Militaire philosophe. On apprend plus $\operatorname{tard}^{29}$ que c'est l'œuvre de Jacques-André Naigeon, l'agent littéraire de Diderot, ou plutôt qu'il l'a 'refait en très grande partie,' sauf le dernier chapitre qui est de son intime ami D'Holbach, sur un manuscrit, dont il ignorait l'auteur, intitulé Difficultés sur la religion proposées au père Malebranche. La bibliothèque Mazarine conserve sous ce titre un manuscrit de quatre cent quatre vingt une pages grand in quarto. ${ }^{30}$ On sait maintenant que ce très original traité déiste écrit vers 1710 est de Robert Challe. Il est composé de quatre 'cahiers.' Le premier contient, dit l'auteur, 'ce qui m'a fait ouvrir les yeux.' Le deuxième, divisé en vingt et une vérités, s'intitule 'examen général des religions factices.' L'imprimé de Naigeon comporte une introduction, dix-neuf chapitres qui remanient copieusement ces deux premiers cahiers, et un vingtième chapitre où $\mathrm{D}^{\prime}$ Holbach a tout ajouté de son cru. La conclusion du deuxième cahier, 'réfutation de la foi,' le troisième cahier, consacré à l'examen de la religion chrétienne, et le quatrième cahier, le moins conformiste, 'contenant un système de religion fondé métaphysiquement sur les lumières naturelles et non sur des faits' ont tout simplement disparu de l'imprimé. Six ans après la trahison déiste de Meslier par Voltaire, Naigeon fait subir une manipulation matérialiste et athée à la plus belle expression du déisme militant. Entre 1760 et 1770, les éditions plus ou moins remaniées n'ont pas manqué et un savant allemand, Rudolf Brummer, ${ }^{31}$ a étudié en 1932 les publications hollandaises de Naigeon, qui était plus bibliophile qu'écrivain. On y trouve une quantité de manuscrits philosophiques clandestins. 
Que prouvent ces deux emprunts très spectaculaires à Meslier et à Challe? Que Lanson et Wade ont absolument raison au moins sur deux points. Premièrement les manuscrits philosophiques clandestins du début du siècle fournissent toute prête la matière des deux courants d'incrédulité, athée et déiste. Deuxièmement les philosophes des Lumières n'avaient plus qu'à s'y servir et ils l'ont fait. On pourrait multiplier les exemples de ce type. Je voudrais maintenant essayer de montrer que ce passage du manuscrit à l'imprimé n'est que la part la plus visible mais la moins importante de sa contribution au progrès intellectuel des Lumières.

Regarder le manuscrit dans sa projection imprimée, qu'elle soit gravement infidèle, comme dans les deux cas étudiés qui sont des exceptions, ou fidèles sur l'essentiel, comme dans le passage de la dissertation du 'philosophe' à l'article 'philosophe' de l'Encyclopédie et comme dans la plupart des cas d'éditions, c'est le considérer négativement, le réduire à l'état d'un inachevé, d'un inédit, d'un ouvrage imparfait. On reste enfermé dans une interprétation soumise à la ligne de partage du milieu du siècle. Cela revient à dire que tous les manuscrits de la première moitié du siècle n'attendaient que le moment d'être imprimés. D'abord ce serait absurde. Comment imaginer tant $d$ 'auteurs, anonymes ou non, à l'affût d'un relâchement de la surveillance, d'un moment propice? Et surtout c'est oublier les manuscrits de la seconde moitié, copiés et diffusés alors que les imprimés prospéraient.

Si Voltaire et Naigeon n'avaient pas publié le Mémoire de Meslier et Le Militaire philosophe, qu'y aurait-il de réellement changé en dehors de la célébrité, un peu mythique dans les deux cas, du curé et de l'anonyme, entretenue par les troupes militantes des années soixante ralliées au cri de Voltaire: 'écrasons l'infâme?' Rien du point de vue essentiel, c'est-àdire du point de vue de leur carrière manuscrite: on a des preuves certaines qu'ils ont tous deux circulé avant d'être publiés, en copies intégrales et en copies abrégées. ${ }^{32}$ Il faut donc plutôt considérer les éditions tardives de manuscrits précoces comme des accidents de l'histoire et envisager les quelque deux cents manuscrits et leurs milliers de copies, d'un bout à l'autre du siècle sans distinction chronologique, dans leur état d'origine, et étudier leur devenir manuscrit. On découvre alors une fantastique activité d'élaboration des idées. Emprunts, variantes, additions, corrections, retranchements, substitutions, interpolations, changements de plan, fabrication d'extraits, de résumés, d'abrégés, associations en recueils, échanges de titres, commentaires, lettres d'accompagnement, contaminations, les ressources insoupçonnables $\mathrm{du}$ manuscrit sont les innombrables gages d'une créativité individuelle et collective inouïe. 
Une des particularités de ce vaste champ littéraire encore mal défriché est en effet d'être à la fois un phénomène collectif et l'expression de fortes personnalités. Un homme, Benoît de Maillet (1656-1738), et un texte, communément appelé Telliamed, symbolisent cette fascinante dualité. Par une abondante correspondance on sait que cet ancien consul général d'Egypte a passé au moins trente ans de sa vie partagé entre le désir frénétique de publier son Nouveau Système du monde et le besoin quasi maladif de le perfectionner avant l'édition. Il n'est pas question ici de la crainte de la censure et des poursuites mais seulement du jugement du public. Dans ce perpétuel souci d'amélioration et ne se fiant pas à son style, Maillet sollicite l'aide de divers correspondants, de Fontenelle même, à qui il demande par surcroît de lui fournir un jeune homme de talent pour 'habiller' son traité. Il est finalement mort sans avoir réalisé son rêve. ${ }^{33}$ Cet exemple est significatif de la différence que faisaient les auteurs eux-mêmes entre la diffusion de l'imprimé et la diffusion du manuscrit. Maillet développe contre la Genèse un système très original pour lequel il se passionne jusqu'à l'exaltation et qu'il ne trouve jamais assez parfait pour l'édition, c'est-à-dire pour le donner à lire par le circuit ordinaire de l'imprimé, mais pendant qu'il le retouche à l'infini, il le fait circuler sous toutes les formes provisoires qu'il revêt successivement. Maillet avait une écriture illisible, il utilisait presque toujours des secrétaires pour sa correspondance, il n'est donc pas surprenant qu'aucun des douze manuscrits que nous connaissons à l'heure actuelle ne soit de sa main. Mais ils permettent de suivre avec une précision exceptionnelle les états du texte qui se sont succédé pendant trente ans sous le contrôle de Maillet mais avec des plumes étrangères. Un manuscrit de travail que j'ai découvert en 1981 à la Bibliothèque Municipale de Vire dans les papiers de Thomas Pichon (1700-1781) nous livre même la méthode suivie. ${ }^{34}$ Sa présentation synoptique de deux versions très différentes juxtaposées ou interlignées, couvertes de ratures et enrichies d'additions marginales et de feuilles volantes élucide presque tous les problèmes que posaient jusqu'alors les différentes familles de manuscrits du Telliamed sous des titres divers. Pour l'anecdote je ne citerai qu'un des accidents pittoresques de transcription. Une bévue de copiste trouve son origine dans le manuscrit de Vire qui est rempli d'abréviations. Deux de ces abréviations se ressemblent beaucoup, ce sont celles de 'partie(s)' et de 'philosophe(s).' Une copie conservée à la Bibliothèque Nationale diffère de toutes les autres par une phrase bizarre: 'si les hommes étoient asses nombreux et assez forts et perseverans pour vouloir en l'état present transporter des philosophes terrestres du septentrion dans les mers meridionales en assez grande quantité....' C'est superbe mais ce n'est qu'une mauvaise lecture de l'abréviation du manuscrit de Pichon qui signifiait plus platement 'parties terrestres'! 
Ce détail cocasse ne nous détourne pas de l'essentiel. Il signifie en effet qu'il n'y a guère de fantaisies de copistes et que les divergences insignifiantes d'une copie à l'autre ont en général une explication banale de ce type. Cette précision est nécessaire pour faire mieux ressortir en revanche l'importance des vraies variantes significatives d'une version à l'autre, dont le rôle est essentiel dans l'ensemble de notre corpus. Elles ne relèvent pas de l'initiative fortuite d'un copiste mais d'une volonté déterminée de remaniement. Cette volonté peut être celle de l'auteur primitif, c'est le cas de Maillet, on vient de le voir. Elle peut être aussi la volonté d'un réviseur. Et tous les cas de figure sont possibles dans les relations entre ces deux types de remaniement, introduit par l'auteur ou introduit par un tiers. Elles peuvent cohabiter dans une même version ou fonder des versions divergentes. Les effets textuels de ce type de transformation varient énormément. Ils vont de la correction de style à la transformation profonde.

La comparaison des copies des Difficultés sur la religion révèle un beau cas de révision du premier type. La langue de Robert Challe (1659-1721) telle que nous la connaissons par son Journal de voyage, ses Mémoires, son roman, est pittoresque, imagée, archaïque et remplie de traits personnels. La première copie intégrale connue, celle de la Mazarine, présente un style dans l'ensemble plus moderne, dépouillé, peu remarquable. La différence était assez sensible pour fournir quelques arguments aux adversaires de l'attribution des Difficultés à l'auteur des Illustres Françaises. La consultation des extraits conservés à Saint-Pétersbourg apporte la preuve qu'ils ont été tirés d'une version antérieure: ils sont remplis de tournures typiquement challiennes et d'allusions biographiques incompréhensibles pour un lecteur qui ne connaît pas les autres oeuvres de Challe ou qui plus simplement ne peut pas penser à faire le rapprochement puisque tous ses ouvrages sont anonymes au XVIIIe siècle. On a alors la preuve qu'entre la version intégrale ancienne d'où ils ont été tirés et la version intégrale de la Mazarine, il y a eu une révision qui a consisté en allégements, corrections conformistes, suppressions des marques d'individualité. Une copie intégrale découverte à Munich en $1991^{35}$ présente un état encore plus ancien et plus fidèle à Challe que les extraits de Saint-Pétersbourg et prouve qu'il y a eu non pas une mais deux révisions dans le sens de ce qui est à nos yeux un appauvrissement. De telles modifications qui portent apparemment sur l'expression, un mot changé ici, là un paragraphe allégé, un autre supprimé ailleurs, finissent en réalité par infléchir la signification du texte.

J'ai parlé de 'réviseur'; c'est un mot vague pour désigner un anonyme, pas plus anonyme d'ailleurs que la plupart des auteurs originels, qui intervient à un moment de la chaîne de circulation du manuscrit, et effectue des modifications qui vont de l'amendement stylistique, de son 
point de vue du moins, à la transformation idéologique. Son statut n'est pas toujours clair. Il s'agit en somme d'un lecteur qui se transforme, spontanément ou non, en relecteur et correcteur, et qui recopie ou fait recopier une nouvelle version, avec petites corrections ou grands bouleversements. Ce lecteur peut être un simple particulier, un auteur qui se livre à cet exercice pour sa satisfaction intellectuelle, un auteur qui prépare le texte pour une édition de son cru. Le rôle de Voltaire dans les extraits manuscrits de Meslier, par exemple, n'est encore ni prouvé ni démenti.

Il est fréquent qu'un manuscrit rencontre plusieurs fois dans sa carrière des lecteurs de ce type. Son message se transforme, peu à peu ou brusquement, son histoire se complique, ses rejetons circulent à leur tour, créent des familles qui lui échappent à leur tour. Il arrive fréquemment, dès 1740 mais surtout après 1760 et souvent dans la fabrique de Ferney, qu'un imprimé tiré de l'un ou de l'autre de ces états manuscrits se mette aussi dans le circuit, sous sa forme imprimée puis en copies manuscrites. L'Examen de la religion court sous plus de dix titres et sa circulation est attestée dès $1720 .^{36}$ Nous en connaissons soixante-dix copies qui se répartissent grossièrement en trois grandes familles dont l'archétype remonte à 1705 et à l'intérieur desquelles il y a des versions différentes, qui elles-mêmes présentent des variantes. Le nombre des chapitres varie de cinq à dix-sept mais les trois répartitions les plus courantes sont onze, quatorze et quinze chapitres. Sans entrer dans les détails d'érudition qui seraient nécessaires pour rendre compte des remaniements idéologiques, on se contentera très schématiquement de signaler une inspiration déiste affirmée, avec profession de foi et prière 'au créateur et souverain maître,' une tendance légèrement matérialiste et une tendance spinoziste. On ajoutera que le dernier chapitre est identique dans les familles à onze et quinze chapitres mais qu'il est manquant dans certaines copies rattachées à ces familles et qu'il constitue la plus grande partie d'un manuscrit unique intitulé De la conduite qu'un honnête homme doit garder pendant sa vie. Une version de la famille en quinze chapitres est le résultat d'une fusion avec un tout autre manuscrit intitulé L'Esprit de Spinoza. Sous ce titre circule une des versions du Traité des trois imposteurs dont nous connaissons une centaine de copies réparties en quatre familles principales entre lesquelles les échanges et les variations sont encore plus complexes. Il faut ajouter que La religion chrétienne analysée, manuscrit en circulation depuis 1735 ou 1740 dont il reste plus de vingt copies, reprend presque intégralement des morceaux de l'Examen de la religion. Pour donner une idée de l'imbrication des traités, de leur parentés externes, précisons qu'il ne faut pas confondre ce dernier avec un traité représenté actuellement par huit copies, intitulé: De l'Examen de la religion qui circule depuis les années 
trente et qui constitue les deux derniers chapitres de l'Examen critique des apologistes de la religion chrétienne, lui-même connu par neuf copies.

Cet aperçu trop rapide des métamorphoses textuelles que peut engendrer la circulation manuscrite au XVIIIe siècle ne porte que sur sept traités, représentant à eux sept plus de deux cents copies. Ce n'est qu'un échantillon des deux cents traités répertoriés et de leurs milliers de copies. On y découvre un formidable chantier intellectuel, transformable à l'infini, totalement ouvert à la liberté d'expression et d'endoctrinement, à l'expérimentation des idées et à leur reproduction, à l'essai des systèmes et à leur diffusion, à la critique individuelle et collective, à la correction spontanée, à la contribution des intelligences, à la répercussion des savoirs, où s'inventent un nouveau mode d'écriture et un nouveau mode de lecture totalement interchangeables. Le manuscrit ainsi exploité, qu'il soit originellement anonyme ou non, est une forme vivante en continuel devenir, une matière malléable offerte à tous, une invitation à collaborer. Au début du siècle l'invitation s'adressait silencieusement entre gens sûrs et discrets, esprits cultivés de la même chapelle intellectuelle. Ils publiaient sous leur noms des traductions, des traités de grammaire, des ouvrages savants. Ils n'avaient pas besoin de publicité bruyante ni de satisfaction supplémentaire d'auteur pour ces essais manuscrits sur le monde, l'âme et Dieu, qu'ils se donnaient à lire, à copier, à commenter. C'étaient des hommes de leur temps. Leurs préoccupations intellectuelles n'étaient ni isolées ni originales. Leurs écrits répondaient à des interrogations de plus en plus répandues, ils ont circulé de plus en plus largement, en se transformant de plus en plus. La méthode faisait ses preuves, la production s'est intensifiée. Pourquoi alors aurait-on renoncé, au profit de l'impression, à une méthode de diffusion d'une telle efficacité? Pourquoi ne pas plutôt laisser se développer un mouvement si bien lancé avec, et non pas contre, d'autres moyens de diffusion? Au cours du siècle, l'évolution des mentalités, de la librairie, de l'imprimerie, loin de tarir la source manuscrite, l'ont alimentée comme elle-même les avaient inspirées. Il n'y a pas de contradiction entre les moyens, pas de scission à un moment du siècle.

Concluons. La transformation anonyme effectuée sur des dizaines de traités dans leur parcours manuscrit, au fil du temps, au gré des lectures, des circuits de colportage et des courants idéologiques ne constitue pas un phénomène conjoncturel, limité à quelques années de pénurie d'imprimé et de liberté, mais un phénomène durable et capital dans l'histoire des Lumières. Le manuscrit philosophique clandestin est un livre, pas un livre inachevé en attente de publication, pas non plus un livre figé. L'auteur d'un livre imprimé, s'il veut le corriger ou le compléter, doit mettre en branle une nouvelle édition. Le lecteur d'un livre imprimé peut le critiquer, le commenter, le développer dans les marges et en sur- 
charges de toutes sortes, ce que les hommes du XVIIIe siècle pratiquaient volontiers, il reste le livre d'un autre. Le manuscrit philosophique clandestin du XVIIIe siècle est un livre vivant, un livre interactif. Qu'il soit relié, doré sur tranches, orné d'un ex-libris, admirablement calligraphié, ou qu'il tienne avec des rubans et qu'il soit rempli de ratures et de paperolles, il ménage au lecteur un espace blanc infini. Il suffit de recopier à sa façon le livre lu, en l'abrégeant ou en le développant, en coupant un paragraphe ou un chapitre, pour le supprimer ou pour le 'coller' ailleurs. La technique est toute prête, elle fonctionne sur une grande échelle. L'histoire des religions, la métaphysique, l'exégèse ne sont pas réservées au savant, au spécialiste, à celui qui est introduit auprès des libraires, elles sont à la portée de n'importe quel lecteur. Le manuscrit rapproche le papier de l'esprit, sans intermédiaire. Il est un message, une incitation, il favorise le dialogue, le progrès de la pensée humaine, l'élaboration d'une pensée collective, la remise en question permanente. Loin d'être un instrument du passé, ce qu'il paraît être si on l'oppose à l'imprimé, il est un instrument de la modernisation des idées, de leur adaptation, de leur pénétration dans les esprits. Le manuscrit n'est pas un pis-aller, il est une tactique. Il n'est pas seulement un outil de diffusion, il est l'outil majeur du progrès de la pensée des Lumières.

\section{GENEVIÈVE ARTIGAS-MENANT UMR 8599 Paris IV-CNRS}

\section{Notes}

1 Extrait de la légende du frontispice du Dictionnaire des sciences, des arts et des métiers, gravé par Charles-Nicolas Cochin en 1764.

2 Voltaire, lettre à Damilaville du 21 décembre 1763, Correspondence, éd. déf. Th. Besterman, D 11577 (Oxford: Voltaire Foundation, 1971-77); traduction et adaptation par F. Deloffre, Correspondance (Paris: Gallimard, Bibliothèque de la Pléiade, 1977-93), t. VII, p. 479.

3 Dictionnaire universel français et latin...[dit de Trévoux], (Paris: Libraires associés, 1752), t. V.

4 Voir à ce sujet De bonne main. La communication manuscrite au XVIIle siècle (Actes de la Table ronde de Bristol, juillet 1991), éd. F. Moureau (Paris \& Oxford, 1993), passim et en particulier F. Moureau, 'La plume et le plomb'; voir aussi R. Darnton, 'Du libertinage aux Lumières,' Tendances actuelles dans la recherche sur les clandestins à l'âge classique, Actes de la journée de Créteil, La lettre clandestine, $\mathrm{n}^{\circ} 5$ (1996): 157-60. 
5 Gustave Lanson, 'Questions diverses sur l'histoire de l'esprit philosophique en France avant 1750,' Revue d'Histoire littéraire de la France (RHLF), 19 (1912): 1-29, 293-317.

6 Catalogue général des manuscrits des bibliothèques publiques de France. 82 vols (Paris, 1886-1935), in $8^{\circ}$.

7 A l'Arsenal, la Mazarine, la Bibliothèque Nationale et dans les bibliothèques municipales de Châlons-sur-Marne, Douai, Fécamp, Rouen.

8 Mémoire des pensées et sentiments de Jean Meslier... , B.N. fr 19458, 19459, 19460; Arsenal 2237, 2559; Fécamp 17-18; manuscrit trouvé à la mort de Meslier (1664-1729). Voltaire en a fait deux éditions très abrégées sous le titre d'Extrait et de Testament (Genève, 1762). Voir Meslier, Oeuvres , 3 vols, éd. J. Deprun, R. Desné, R. Soboul (Paris: Anthropos, 1970).

9 Le Ciel ouvert à tous les hommes, ou traité théologique dans lequel, sans rien déranger des preuves de la religion, on prouve solidement, par l'ecriture sainte et la raison, que tous les hommes sont sauvés, B.N. fr 9619, 9620, 17106, 20109, 22925, 22926; Arsenal 2238, 2560; Mazarine 1176, 1177. Première édition en 1768. Voir P. Cristofolini, Il Cielo aperto di Pierre Cuppé (Florence: Leo S. Olschki, 1981).

10 Ce titre est celui de l'édition de Naigeon (1768) d'un extrait très abrégé d'un manuscrit intitulé Difficultés sur la religion proposées au Père Malebranche, Mazarine 1163. Voir R. Challe, Difficultés sur la religion, éd. F. Deloffre (Oxford, 1982).

11 Sans doute par l'influence du titre de l'ouvrage de Wade, voir ci-dessus note 14 .

12 Lanson, op. cit.: 2-3.

13 Lanson, op. cit. p.3.

14 Ira O. Wade, The Clandestine Organization and Diffusion of Philosophic Ideas in France from 1700 to 1750 (Princeton UP, 1938; rééd. New York: Octagon Books, 1967).

15 Daniel Mornet, La Pensée française au XVIIIe siècle (Paris, 1929); du même auteur, Les Origines intellectuelles de la Révolution française: 1715-1789 (Paris, 1933); P. Hazard, La Crise de la conscience européenne: 1680-1715. 3 vols (Paris, 1935).

16 Miguel Benítez, 'Liste et localisation des traités clandestins,' contribution d'abord présentée à la Table Ronde de 1980 puis publiée dans Le Matérialisme du XVIIIe siècle et la littérature clandestine, sous la direction d'Olivier Bloch (Paris: Vrin, 1982), pp. 17-25; du même auteur, 'Matériaux pour un inventaire des manuscrits philosophiques clandestins des XVIIle et XVIIIe siècles,' Rivista di storia della filosofia, ${ }^{\circ} 3$ (Milan: Franco Angeli, 1988):501-20; du même auteur, La Face cachée des Lumières (Paris: Universitas \& Oxford: Voltaire Foundation, 1996), pp. 20-54.

17 Sous la direction d'Olivier Bloch et Antony McKenna, elle est publiée par l'UMR 8599 de Paris IV, le C.H.S.P.M. de Paris I, l'UMR 5037 de Saint-Etienne, et, depuis 1995, l'EA 431 de Paris XII. Alain Mothu rassemble et met à jour toutes les informations qui lui sont communiquées. $\mathrm{N}^{\mathrm{O}} \mathrm{s} 1$ à 4 sur Internet: http:/ / lancelot.univ-paris12.fr/arb-publi.htm ; ns 5 à 8 (Paris: Presses de l'Université de Paris-Sorbonne).

18 Ce développement a suscité la création d'un Comité d'initiative pour l'Inventaire des manuscrits philosophiques clandestins des XVIIe et XVIIIe siècles qui s'est constitué les 4 et 5 juin 1986. Il est composé de Miguel Benítez (Séville), Olivier Bloch (Paris), Guido Canziani (Milan), Gianni Paganini (Milan), Jeroom Vercruysse (Bruxelles). Son siège a été fixé à Milan, au Centro di Studi del Pensiero filosofico del Cinquecento e del Seicento du C.N.R. italien. 
19 Fondée par Olivier Bloch, professeur d'histoire de la philosophie à l'Université de Paris I - Panthéon Sorbonne et actuellement dirigée par Geneviève Artigas-Menant, cette équipe fait partie du Centre d'étude de la langue et de la littérature françaises des XVIIle et XVIIIe siècles de Paris IV-Sorbonne (CELLF 17-18), UMR 8599 du CNRS.

20 The Romanic Review, XXX (1939): 205-09.

21 Voir ci-dessus, note 2.

22 Voir dans Tendances actuelles dans la recherche sur les clandestins à l'Age classique, La Lettre clandestine $\mathrm{n}^{\circ} 5$ (1996), les articles de Véronique Sarrazin 'Du bon usage de la censure au XVIIIle siècle' et de Frédéric Barbier 'Sur les routes de l'interdit: espaces et réseaux du livre de contrebande entre le Nord et Paris au XVIIIe siècle.' Voir aussi dans Censure et clandestinité aux XVIIe et XVIIIe siècles, La Lettre clandestine $\mathrm{n}^{\circ} 6$ (1997), les articles de Sabine Juratic, 'Commerce et réseaux du livre clandestin au XVIIIe siècle'; Dominique Varry, 'Le livre clandestin à Lyon au XVIIIe siècle'; Edoardo Tortarolo, 'La censure à Berlin au XVIIIe siècle'; Françoise Weil, 'Les livres persécutés en France de 1720 à 1770'; François Moureau, 'Du clandestin et de son bon usage au XVIIIe siècle.'

23 Mémoires pour l'histoire des Sciences et des Beaux-Arts, imprimé à Trévoux, vendu à Paris (mars 1705), 2ème partie, t. I, p. 545.

24 Ibid., (août 1743), pp. 2267-2294.

25 Pour une analyse détaillée de ce cas passionnant, voir l'édition d'Olivier Bloch, Parité de la vie et de la mort, La Réponse du médecin Gaultier (Paris: Universitas \& Oxford: Voltaire Foundation, 1993).

26 Lanson, op. cit.: 3.

27 Extrait des sentimens de Jean Meslier adressés à ses paroissiens, sur une partie des abus et des erreurs en général et en particulier (Genève, 1762) et Testament de Jean Meslier. Nouvelle édition (Genève, 1762). Pour le détail des différentes éditions voir J. Deprun, R. Desné, A. Soboul, CEuvres de Jean Meslier (Paris: Anthropos, 1970), t. III, pp. 581-89.

28 BN f.f. 19458, 19459, 19460; 311, 333, 358 ff. 175x130 mm.

29 Barbier, Dictionnaire des ouvrages anonymes et pseudonymes, 2ème éd. (1822).

30 Mazarine 1163. C'est ce manuscrit que Roland Mortier prend comme texte de base de son édition anonyme, Difficultés sur la religion (Presses universitaires de Bruxelles, 1970).

31 Rudolf Brummer,Studien zur französischen Aufklärungsliteratur im Anschluss an Jacques-André Naigeon (Breslau, 1932).

32 Nous connaissons huit copies intégrales du Mémoire, en plus des trois de la main de Meslier, et deux desDifficultés. Nous connaissons trois exemplaires abrégés ou extraits des Difficultés et vingt-et-un du Mémoire.

33 Fritz Neubert, rédigeant une Introduction à une édition critique de Telliamed (Einleitung in eine kritische Ausgabe von B. de Maillets Telliamed, Romanische Studien, Heft 19 [Berlin, 1920], 215p.), en dénombre cinq copies dont deux à la Bibliothèque Nationale et les autres à l'Arsenal et dans les Bibliothèques municipales de Chartres et du Mans (Chartres 762, Le Mans 384, Paris: Arsenal 2885, BN f.fr. 9774, f.fr. 9775). L'édition de Fritz Neubert n'a jamais été faite. Il y a eu 3 éditions au XVIIIe siècle: Telliamed, ou Entretiens d'un philosophe indien avec un 
missionnaire françois sur la diminution de la mer, la formation de la terre, l'origine de $l^{\prime} h o m m e ~ e t c$. Mis en ordre sur les Mémoires de feu M. de Maillet par J.A.G*** [Guer], 2 vols in $8^{\circ}$ (Amsterdam, 1748); 2nde édition (Basle: chez les libraires, 1749); 3ème édition, dite 'Nouvelle édition, revue, corrigée et augmentée sur les originaux de l'auteur, avec une vie de M. de Maillet' [par Le Mascrier] (La Haye, 1755). L'édition de la collection 'Corpus' (Fayard, 1984) reproduit l'édition de 1755 avec les pages, mais en modernisant la typographie.

34 Vire C 828.

35 Par François Moureau (Munich: Bayerische Staatsbibliothek), Cod. Gall. 887.

36 A. Thomson et $\mathrm{F}$. Weil, 'Manuscrits et éditions de l'Examen de la religion,' Le Matérialisme du XVIIIe siècle et la littérature clandestine, éd. O. Bloch (Paris: Vrin, 1982), pp. 177-85; A. Thomson, 'L'Examen de la religion,' Filosofia e religione nella letteratura clandestina, éd. G. Canziani (Milan: FrancoAngeli); G. Mori, 'L'Examen de la religion au XVIIIe siècle,' Censure et clandestinité aux XVIIe et XVIIIe siècles, dans La Lettre clandestine, $\mathrm{n}^{\circ} 6$, (1997): 201-28. 